\title{
A eficiência da materialidade. $O$ recurso a ferramentas digitais de simulação e fabricação aditiva na procura de uma maior eficácia dos dispositivos
}

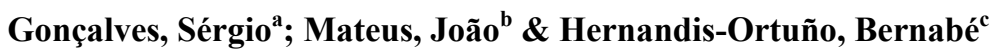 \\ ${ }^{a}$ Escola Superior de Artes e Design de Caldas da Rainha, Instituto Politécnico de Leiria, Laboratório de investigação \\ em Artes e Design, Centro de Desnvolvimiento Rápido e Sustentado de Produto, Portugal. \\ sergio.goncalves@ipleiria.pt \\ ${ }^{\mathrm{b}}$ Escola Superior de Artes e Design de Caldas da Rainha, Instituto Politécnico de Leiria, Laboratório de investigação \\ em Artes e Design, Centro de Desnvolvimiento Rápido e Sustentado de Produto, Portugal. sergio.goncalves@ipleiria.pt \\ jvmateus@ipleiria.pt \\ ${ }^{c}$ PhD Full Professor - Universitat Politècnica de València, Spain. bhernand@upv.es.
}

\begin{abstract}
Resumo
A praxis do Design de produtos, condicionada pelas crises sociais e ambientais que o mundo atravessa, deve passar pela procura de uma cada vez maior eficiência dos objetos na sua dimensão ambiental. Embora esta questão esteja a jusante de questões eventualmente mais relevantes na minimização deste problema, e que têm a ver com a legitimação da própria existência dos dispositivos, a otimização do recurso a matériasprimas no fabrico de objetos é de fulcral importância. A possibilidade de otimizar os produtos pela redução da incorporação de matéria-prima no seu fabrico e adequar o material a uma determinada utilização é central no seu processo de desenvolvimento. Por outro lado, as novas possibilidades trazidas pela fabricação aditiva no sentido de reduzir a quantidade de materiais e processos industriais associados à produção de componentes técnicos complexos permitem perspetivar uma melhoria significativa do impacto ambiental tradicionalmente associado à sua obtenção.
\end{abstract}

O presente artigo procura examinar como as tecnologias digitais de análise, otimização e fabricação aditiva poderão constituir uma resposta viável para uma produção mais eficiente do ponto de vista da incorporação de recursos não-renováveis e da minimização do impacto ambiental associado às tecnologias de produção tradicionais.

Será efetuada uma pesquisa bibliográfica relativa à questão da sustentabilidade e tecnologias de fabricação aditiva, assim como às suas relações com o design.

Serão seguidamente identificados exemplos que testemunhem o estado atual da aplicabilidade destas tecnologias na produção direta de objetos, a partir dos quais se procurarão tirar conclusões que permitam entender em que medida as tecnologias aditivas poderão ter um impacto positivo na sustentabilidade ambiental, e como poderá o design posicionar-se perante esta nova realidade.

Palavras chave: design, sustentabilidade, fabricação aditiva. 
A eficiência da materialidade. O recurso a ferramentas digitais de simulação e fabricação aditiva na procura de uma maior eficácia dos dispositivos.

\begin{abstract}
The praxis of product design, conditioned by social and environmental crises that the world is facing, must go through the search for increasing efficiency of objects in its environmental dimension. Although this issue is downstream from possibly more relevant issues to minimize this problem, and that have to do with the legitimacy of the existence of the devices themselves, optimizing the use of raw materials in the manufacture of objects is of crucial importance. The ability to optimize products by reducing the incorporation of raw materials in their manufacture and adapting the material for a particular purpose, is central to the development process. Moreover, new possibilities brought by the additive manufacturing to reduce the amount of materials and industrial processes associated with the production of complex technical components, allows to foresee a significant improvement in environmental performance traditionally associated with its obtainment.

This paper seeks to examine how digital Technologies of optimization, analysis and additive manufacturing, may constitute a viable response to a more efficient production from the point of view of the incorporation of non-renewable resources and minimize the environmental impact associated with traditional production technologies.

A bibliographic research on the issue of sustainability and additive manufacturing technologies, as well as its relations with design will be made.

Examples will then be identified, which witness the current state of the application of these technologies in the direct production of objects, from which the drawing of conclusions will be sought, allowing us to understand to what extent the additive technologies can have a positive impact on environmental sustainability, and how can the design positions itself to this new reality.
\end{abstract}

Keywords: design, sustainability, additive manufacturing

\title{
1. Introdução
}

A problemática ambiental subjacente da atividade humana tem, na atualidade, uma urgência que cresce a cada dia que passa. Passamos a coexistir no quotidiano com os dramáticos impactos da atividade desregulada do mundo ocidental, com consequentes catástrofes ambientais e uma enorme pressão sobre os ecossistemas, a qualidade do ar e da água. A situação atinge proporções dramáticas em países com elevados níveis de produção industrial, como a China, mas também em países pobres como o Gana, apenas para dar dois exemplos que ilustram a capacidade devastadora da atividade humana, independentemente da prosperidade ou situação geográfica dos países. Sendo desde há muito conhecida a realidade de Agbogbloshien no Gana, para onde, segundo o site WorstPolluted.org, são anualmente exportadas 215.000 toneladas de lixo eletrónico (que se prevê poder chegar ao dobro em 2020, no caso de 
se manter a linearidade do crescimento económico), o alarme soou veementemente no final de 2015 com o elevado nível de poluição do ar atingida em Pequim, para onde uma empresa canadiana passou a exportar ar embalado no início de 2016. Segundo o site do jornal The Telegraph, "Cada embalagem de 7,7l é vendida por um montante aproximado de $€ 13$, o que representa um custo 50 vezes maior que uma garrafa de água mineral na China." $\mathrm{O}$ ar passou assim de um bem vital gratuito a um produto transacionável, não por capricho ou excentricidade, mas por necessidade vital. Adensa-se, portanto, com o passar do tempo, a nuvem que paira sobre a humanidade, apesar das preocupações ambientais fazerem parte do debate mundial desde os anos sessenta, fortemente impulsionado pelo livro "Silent Spring" de Rachel Carson, que $\mathrm{Al}$ Gore qualifica como o "registo de nascimento do movimento ecológico".

Nos anos setenta, o design começa a considerar a questão ambiental como tema central da sua atividade. Tomás Maldonado, já em 1970 reconhecia que, "o ambiente humano, um dos muitos subsistemas que compõem o vasto sistema ecológico da natureza é o único subsistema que possui a capacidade virtual e real de provocar perturbações substanciais - isto é-irreversiveis, no equilibrio dos outros subsistemas" (Maldonado, 1970). No ano seguinte, Victor Papanek publica o livro "Design for the real world", obra que se foi tornando referencial, e que já nessa altura alertava para a necessidade de repensar a prática do design, alegando que "Nunca afirmaremos suficientemente que, nos problemas de poluição, o designer está mais implicado que a maioria das pessoas" (Papanek. 1971).

45 anos passaram desde a publicação do livro de Papanek, e apesar de todos os esforços, estratégias, metodologias, campanhas e diferentes abordagens ao design, os resultados são diminutos, e não foram compensando a degradação galopante do ambiente.

A mudança necessária deveria passar por mudanças a vários níveis, a maior parte dos quais sociais e políticos, mas também ao nível da forma como são projetados, fabricados e utilizados os objetos e diferentes dispositivos.

A forma de projetar e produzir está em plena mudança. As tecnologias digitais de modelação evoluíram num curto espaço de tempo, começando pela capacidade de modelar e visualizar objetos em ambiente virtual, seguindo-se a capacidade de imprimir esses modelos a três dimensões. A tecnologia progrediu e atingiu a capacidade real de produzir objetos e componentes mecânicos fiáveis e precisos, de forma direta e em diferentes tipos de materiais. Esta possibilidade coloca o design perante uma forma completamente nova de se relacionar com o fabrico e a difusão dos objetos e obriga-o a entender como se adaptar às novas possibilidades tecnológicas, e sobretudo, entender em que dimensão estas poderão contribuir para minimizar os impactos associados à produção.

\section{A eficiência da materialidade}

Embora se parta do princípio que a questão da sustentabilidade ambiental não se limita às questões relacionadas com a matéria e a sua transformação em objetos, esta é uma dimensão determinante na resolução do problema. A eficiência da materialidade, entendida enquanto qualidade do que é material, é um aspeto incontornável. Este é o foco central da maioria das áreas que se debruçam sobre a sustentabilidade dos objetos na sua dimensão material, nomeadamente o eco design, o cradle-to-cradle ou a economia circular. A redução da quantidade de matéria processada para satisfazer as necessidades das sociedades contemporâneas deverá decrescer substancialmente, de forma a poder encarar-se a possibilidade de um futuro para o planeta.

"Starting from here, while bearing in mind the growing population, as calculated, and the growing demand of the emerging and developing countries, as justified, the third parameter surfaces - 
A eficiência da materialidade. O recurso a ferramentas digitais de simulação e fabricação aditiva na procura de uma maior eficácia dos dispositivos.

technological eco-efficiency - with a rather impressive result: the sustainable requirements are realistic, but only if eco-efficiency is increased 10-fold. In other words: we can consider as sustainable only those production-consumption systems that employ $90 \%$ less material input per unit of service than is actually accounted for in contemporary industrial society." (Vezzoli \& Manzini, 2008)

Manzini conclui que tal implicará um processo de desmaterialização do que a sociedade procura para melhorar a sua qualidade de vida. Esta consistiria numa redução drástica da quantidade de produtos e serviços necessários para uma qualidade de vida socialmente aceitável, acompanhada de um decréscimo colateral dos fluxos de material e energia utilizada, referindo ainda que tal pode ser conseguido através da redução da procura de produtos e serviços incrementando a inteligência do sistema, e diminuindo os seus fluxos de material e energia.

O panorama atual dos produtos de grande consumo continua a basear-se numa forte incorporação de energia e matérias-primas. A forma dos objetos está dependente de constrangimentos tecnológicos que muitas vezes condicionam a sua eficiência, na medida em que constantemente necessitamos de balancear o que se quer fazer com o que a tecnologia permite fazer.

\section{Tecnologias digitais no design de produtos}

\subsection{Considerações gerais}

Há 26 anos, Alan Pipes (Pipes, 1990), escreveu nas primeiras linhas do seu livro que o CAD (Computer Aided Design) poderia alterar a função do designer antevendo a importância que as tecnologias digitais 3D teriam no seu trabalho, e ainda antes de ser patenteada a primeira impressora 3D afirmou que "... the need for prototypes may not be eliminated, but fewer of them will be required and the ones that have to be made will be nearer to the real thing." Anos mais tarde, Chang (Chang et al, 1998) definiu novos princípios orientadores visando a produção rápida do produto com a integração objetiva de metodologias digitais no paradigma de produção DFM (Design for Manufacturing). Também Hague (Hague et al, 2003), referindo-se aos novos processos aditivos de fabricação, propõe o uso destas tecnologias como uma filosofia aplicada de projeto, desde as etapas iniciais do desenvolvimento do produto com o objetivo de projetar artefactos de forma mais flexível e económica e considera que "... This new design freedom will place much more responsibility on the designer to think about exact requirements of a part".

No livro "Digital Design and Manufacturing", Schodek (Schodek et al. 2005) efetua uma análise detalhada das tecnologias digitais aplicadas ao design industrial e destaca a prototipagem rápida e a "freeform fabrication" como ferramentas ideais para o que apelida de "soft tooling", e moldes utilizando metais em pó para produzir objetos de extrema complexidade. Na sequência desta evolução, Ian Gibson (Gibson, 2010) amplia o alcance do DFM para o conceito de emergente DFAM (Design for Additive Manufacturing). Considerando a possibilidade dos designers ignorarem os constrangimentos inerentes a processos tradicionais de produção afirma: "... maximize product performance through the synthesis of shapes, sizes, hierarchical structures, and material compositions, subject to the capabilities of AM technologies."

Wholers (Wholers, 2011) assume a fabricação aditiva como um processo emergente de produção e acredita que num futuro próximo se tornará a tecnologia de maior utilidade no desenvolvimento e fabrico de produtos:

"Additive manufacturing (AM) is going places that many of us never anticipated. Frankly, I believe we've only seen the tip of the iceberg. The more I explore the future potential of $A M$ and $3 D$ printing 
technology, the more excited I become. I truly believe that AM will develop to become the most useful technology for the development and production of products than any other".

No livro "Fabricated: The new world of 3D printing" Lipson (Lipson et al, 2013) afirma que a impressão 3D já não é uma nova tecnologia dado que há décadas que existem equipamentos a produzir objetos e, segundo o autor "... in the past few years, $3 D$ printing technology has been driven rapidly forward by advances in computer power, new design software, new materials, and the rocket fuel of inovation, the Internet."

\subsection{Fabricação/Produção Aditiva (additive manufacturing)}

Apesar da expressão Impressão 3D (3D Printing) ser utilizada vulgarmente para definir este tipo de tecnologias o comité ASTM (Intenational Committee F42 on Additive Manufacturing Technologies) define a fabricação aditiva como:

"...additive manufacturing as the process of joining materials to make objects from $3 D$ model data, usually layer upon layer, as opposed to subtractive manufacturing methods. Synonyms include additive fabrication, additive processes, additive techniques, additive layer manufacturing, and freeform fabrication."

O termo Impressão 3D, mais específico, é definido pelo comité ASTM 42 como a fabricação de objetos através da deposição de material utilizando um cabeçote de impressão, um bocal ou outra tecnologia de impressão. É normalmente associado a sistemas de baixo custo e equipamentos com capacidades de fabricação aditiva limitada.

A FA é utilizada para construir modelos físicos, protótipos, madres, componentes de ferramentas e produzir diretamente peças em plástico, metal, cerâmica, vidro e materiais compósitos.

\subsection{Processos}

Todos os processos da FA apresentam semelhanças. O conceito base é o mesmo. Partindo de um modelo CAD 3D, a fabricação consiste na junção de materiais em sucessivas camadas. Hopkinson (Hopkinson, 2006) identificou mais de vinte sistemas de prototipagem rápida mas reconhece que parte deles não terão capacidade para fabricar diretamente objetos funcionais. Num estádio anterior da tecnologia, os processos de FA eram divididos em três categorias que dependiam do estado inicial da matéria-prima utilizada. Chua (Chua, 2005) e Volpato (Volpato, 2007), separavam os processos em três grupos: os baseados em líquido, os baseados em sólido e os que utilizavam material pulverizado.

Em janeiro de 2012, o comité ASTM F42 aprovou uma lista, definindo designações e especificações para cada processo, que denominou "Standard Terminology for Additive Manufacturing Technologies":

Extrusão de materiais (material extrusion) - processo aditivo onde o material é seletivamente distribuído através de um bocal ou orifício;

Ejeção de materiais (material jetting) - processo aditivo onde gotículas do material de construção são seletivamente depositadas;

Ejeção de ligante (binder jetting) - processo aditivo onde o líquido ligante é seletivamente depositado para agregar materiais pulverizados;

Laminação em folha (sheet lamination) - processo aditivo onde folhas de material são cortadas e ligadas por camada para formar o objeto;

Foto polimerização em cuba (vat photo polymerization) - processo aditivo onde um líquido depositado numa cuba é seletivamente curado através de luz; 
A eficiência da materialidade. O recurso a ferramentas digitais de simulação e fabricação aditiva na procura de uma maior eficácia dos dispositivos.

Fusão em cama de pó (powder bed fusion) - processo aditivo onde energia térmica funde seletivamente regiões determinadas na cama de pó;

Deposição por energia direcionada (directed energy deposition) - processo aditivo onde um foco de energia térmica é utilizado para fundir materiais que vão derretendo à medida que são depositados.

\subsection{Vantagens da produção aditiva}

O facto destas tecnologias, de uma forma geral produzirem objetos por sobreposição de seç̧ões planas, faz com que se possam obter formas altamente complexas sem a necessidade de se possuir particular conhecimento ou destreza técnica. $\mathrm{O}$ tempo associado à obtenção desta forma é assim drasticamente reduzido, ao mesmo tempo que são possíveis formas outrora impensáveis de serem produzidas com as tecnologias de fabrico convencionais.

A forma complexa ganha viabilidade, uma vez que a sua produção passa a ter um valor de produção semelhante ao da forma simples e, como refere Diegel (Diegel et al, 2010) "... With additive manufacturing, complexity and geometry no longer affect manufacturability."

A possibilidade tecnológica de reduzir os constrangimentos formais a que estão sujeitos os produtos permite caminhar no sentido de dispositivos mais eficientes tecnicamente, e mais duráveis emocionalmente quer pela satisfação técnica que proporcionam quer pela possibilidade de os aproximar mais das reais necessidades e espectativas dos utilizadores.

Apesar dos materiais utilizados nos processos que permitem peças mais perfeitas, dado a sua especificidade e complexidade na produção, apresentarem na generalidade custos elevados, o facto de se utilizar apenas a quantidade necessária para produzir o objeto evita desperdício e, como Alice Rawsthorn menciona "...which is not only beneficial environmentally, but financial too, because it reduces the risk of manufacturers wasting money on superfluous raw material." (Rawsthorn, 2013)

\section{Relação do design com a produção aditiva numa perspetiva de sustentabilidade}

Nas palavras de Nathan Stegall (Stegall. 2006), "The field of design... has become a major focal point for sustainability, which is not surprising since poorly designed industrial systems, products, and buildings can greatly contribute to environmental and social degradation."

Entende-se que neste domínio, nomeadamente em termos da qualidade dos dispositivos e do seu impacto na natureza, as tecnologias de produção aditiva vieram ampliar a possibilidade do design poder produzir objetos mais eficientes, mais duráveis, mais próximos dos utilizadores, e simultaneamente menos nocivos na sua relação com o ambiente. Como Alice Rawsthom conclui "... 3D printing also offers an important opportunity to make progress on the sustainable front." (Rawsthorn, 2013)

Inicialmente, as tecnologias de impressão 3D utilizavam-se unicamente para a produção de modelos de estudo, integrados no processo de desenvolvimento do projeto. Ainda na atualidade, e embora as tecnologias aditivas sejam já utilizadas em várias áreas, nomeadamente na medicina e na indústria aeroespacial, este tipo de tecnologia está muito associada à ideia de modelo e não de produto, na medida em que as tecnologias mais democratizadas (makers) ainda não atingiram um nível de rigor superficial ou uma resistência física adequada à maior parte das utilizações. Por outro lado, os produtos que já se fabricam com recurso a estas tecnologias apresentam custos elevados que não permitem a sua maior difusão, e são essencialmente utilizados em situações específicas em que se mostraram competitivos face às tecnologias até agora utilizadas. Com o seu desenvolvimento, as tecnologias aditivas deixarão de ser 
entendidas como ferramentas de prototipagem para passarem a ser entendidas como ferramentas de produção.

A própria relação do designer com o utilizador poderá mudar, e esta nova forma de relacionamento permitirá uma maior adaptação dos produtos aos utilizadores, mas também a possibilidade de recolher dados sobre o desempenho do objeto de uma forma muito mais direta, na medida em que se reduzem os filtros ou intermediários da informação.

O relatório de 2012 da CSC (Computer Sciences Corporation) (CSC, 2012) aponta já uma substancial diferença nessa relação ${ }^{69}$ que passará por um encurtamento da cadeia, nomeadamente no que se refere à montagem, distribuição, armazenamento e venda. O design poderá nesta medida obter um maior proveito económico, garantindo em simultâneo ao utilizador um menor custo de aquisição e uma maior qualidade e adaptação às suas necessidades específicas.

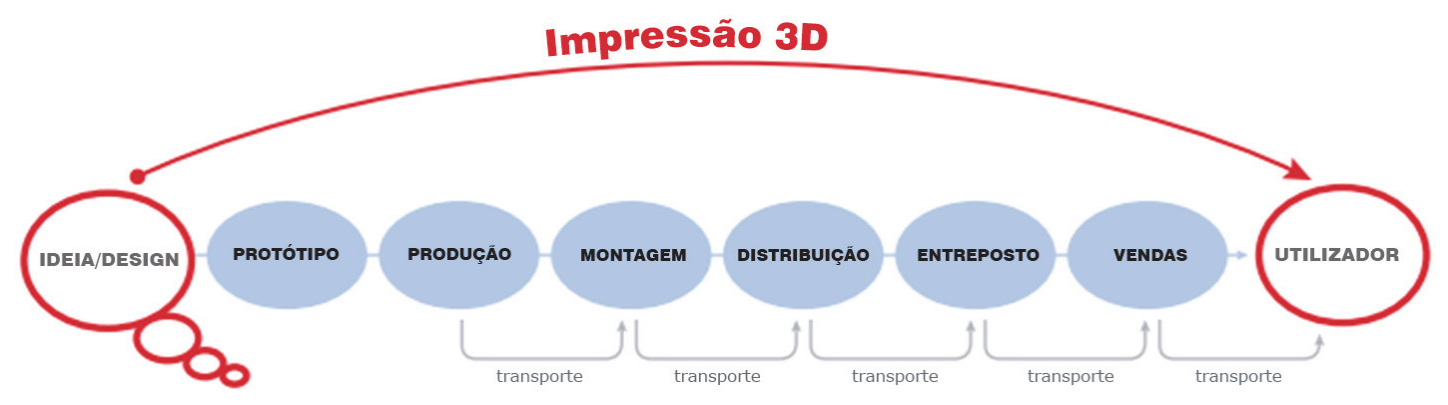

Fig. 1 A oportunidade de longo prazo para particulares (Fonte: Adaptado de (CSC, 2012))

Os produtos dispensarão também o enorme custo económico e ambiental associado ao seu transporte entre as diferentes fases do seu ciclo, e a toda a logística associada.

A adaptação do design e da produção aditiva, numa perspetiva de sustentabilidade ambiental, poderá ser analisada através da sua possibilidade de correspondência a uma estrutura de economia circular, cuja intenção é eliminar o lixo ou a poluição, através de uma relação com os objetos que passa pelo seu uso, cuidado, reparação, reutilização e reciclagem.

\subsection{Adaptação ao utilizador}

Nas economias desenvolvidas, a customização era a norma para ricos e pobres, isto até à revolução industrial, quando a mecanização permitiu produzir grandes quantidades de objetos estandardizados de forma mais eficiente e económica do que a produção convencional (Rawsthorn, 2013).

A produção baseada numa tecnologia que se desliga da necessidade de estandardização, na medida em que a obtenção de formas deixa de estar dependente de moldes ou ferramentas específicas, permitirá estabelecer uma ligação, outrora muito improvável, entre o utilizador e o designer, facilitando fortemente a precisão com que o objeto se adapta à sua utilização específica.

\footnotetext{
${ }^{69}$ Embora o relatório se refira aqui ao "design" como "desenho", na perspetiva de um utilizador poder imprimir diretamente os objetos que desenha na impressora que possui em casa, o diagrama fornece uma imagem clara daquela que pode ser entendida como a realidade da relação entre o designer e o utilizador, assim como as vantagens ambientais daí decorrentes.
} 
A eficiência da materialidade. O recurso a ferramentas digitais de simulação e fabricação aditiva na procura de uma maior eficácia dos dispositivos.

Quer seja através de dispositivos médicos, como aparelhos ou próteses, quer seja através das opções que muitas empresas já disponibilizam (opções estas geralmente limitadas a um leque de escolhas definido), a possibilidade de personalização dos produtos tem vindo a aumentar, o que permite prever que o nível de adaptação terá tendência a acompanhar esta evolução.

Poderemos antever ironicamente um regresso a uma relação entre o utilizador e os produtos semelhante à que prevalecia na pré-industrialização, em que muitos objetos comuns, desde roupa a mobiliário, eram fabricados por medida.

A adaptação ao utilizador é também potenciada pelo facto do protótipo, em rigor, passar a ter o mesmo custo que qualquer um dos seus múltiplos, e o primeiro protótipo validado, mesmo para uma série elevada, pode imediatamente ser vendido e utilizado. Salvo em negociações por escala, as peças de uma pequena série custam exatamente o mesmo que as de uma grande.

Um utilizador, com o mínimo conhecimento informático, passará a relacionar-se muito facilmente com o produtor em plataformas na internet. Provavelmente, tal como se desenvolveu globalmente o serviço de cópia, impressão e digitalização de documentos, também esta tecnologia estará disponível em todas as cidades, e a fabricação de objetos passará a ser local, perto do utilizador.

Como afirma Stegall “....another implication for the form of a product is decentralization: products that make people dependant on large, centralized, distant organizations (current power companies and large power generation plants are prime examples) encourage people to be ignorant of how they work and their environmental impact." (Stegall, 2006)

\subsection{Aumento da vida útil dos produtos}

Uma das dimensões mais interessantes oferecidas pela produção aditiva é a sua capacidade de contribuir para uma maior duração dos produtos. Esta é determinante na perspetiva da sustentabilidade, na medida em que reduz significativamente a necessidade de substituição dos dispositivos por razões de falência técnica ou por razões emocionais.

Em termos de relação emocional, a possibilidade dos produtos serem mais personalizados estabelece relações mais duráveis com os utilizadores. Diegel (Diegel et al. 2010) refere-se a esta questão ao afirmar que "... Designers can stimulate desirability, increase pleasure and deepen attachment by designing products that not only function better, are more aesthetically pleasing than comparable products, but are also tailored to better suit the individual needs of the user."

$\mathrm{O}$ aspeto mais interessante consiste no fato das tecnologias de fabricação aditiva poderem contribuir para aumentar a vida útil dos produtos já existentes. Frequentemente somos confrontados com pequenas avarias, que implicam a substituição de uma peça. Não menos frequente é essa peça já não existir no mercado, ou implicar um custo de tal maneira elevado que torna mais económica a substituição do objeto todo por um novo. Em muitos casos, seria possível prolongar consideravelmente o tempo de vida do objeto, se fosse possível reproduzi-la de maneira económica. Este problema pode ser perfeitamente ultrapassado recorrendo à realização da peça através de produção aditiva. 


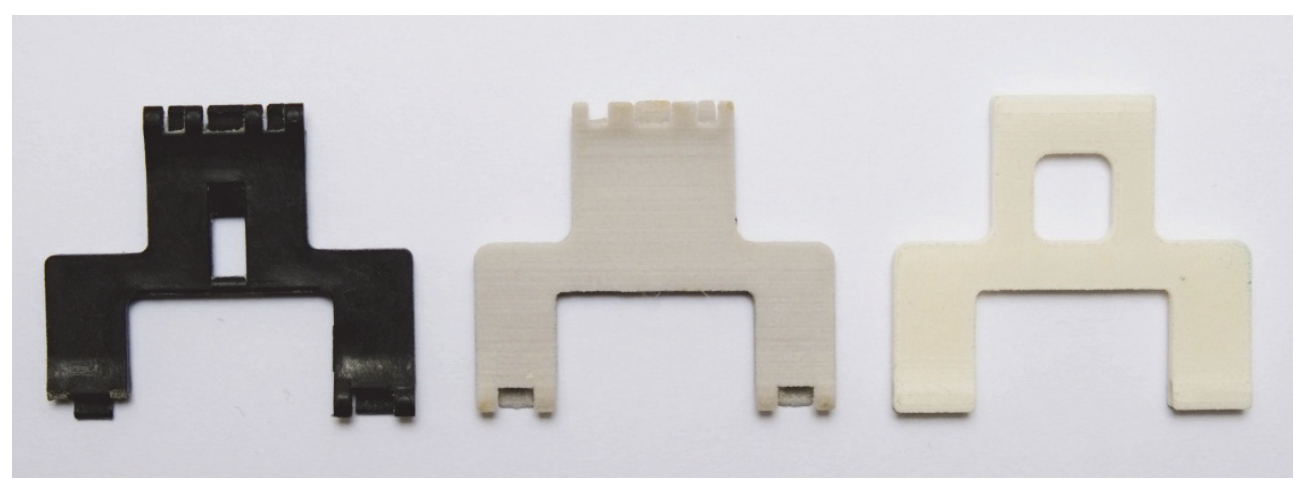

Fig. 2 Peça recuperada de uma impressora 3 D - ZPRINTER

A figura 2 mostra uma peça danificada de uma impressora 3D ZPRINTER, originalmente fabricada por injeção de polímero, e duas fases da sua reprodução, a primeira, na mesma máquina e a segunda, otimizada e mais resistente onde foi usada a tecnologia SLS (Selective Laser Sintering). No primeiro caso, procedeu-se à replicação mimética da peça, nomeadamente no que diz respeito aos encaixes onde passa um eixo de rotação. No segundo caso, foi possível corrigir facilmente o problema de fragilidade desses encaixes, completando a superfície cilíndrica. Neste caso, obtemos um produto final com uma maior fiabilidade, na medida em que foi produzido por uma tecnologia que não limita a forma. No caso da peça original, a forma estava condicionada pela tecnologia de produção, que obriga à supressão de partes simétricas do corpo cilíndrico para permitir a abertura do molde. Acerca desta intervenção, pode referir-se que comparativamente, a peça representou um custo de reparação 100 vezes inferior ao que custaria a reparação com componentes originais.

Este exemplo permite apontar as diversas vantagens do processo:

1 - Rapidez na substituição da peça;

2 - Prolongamento do tempo de utilização da máquina;

3 - Redução dos constrangimentos produtivos que deram origem à peça, possibilitando assim otimizar o seu funcionamento.

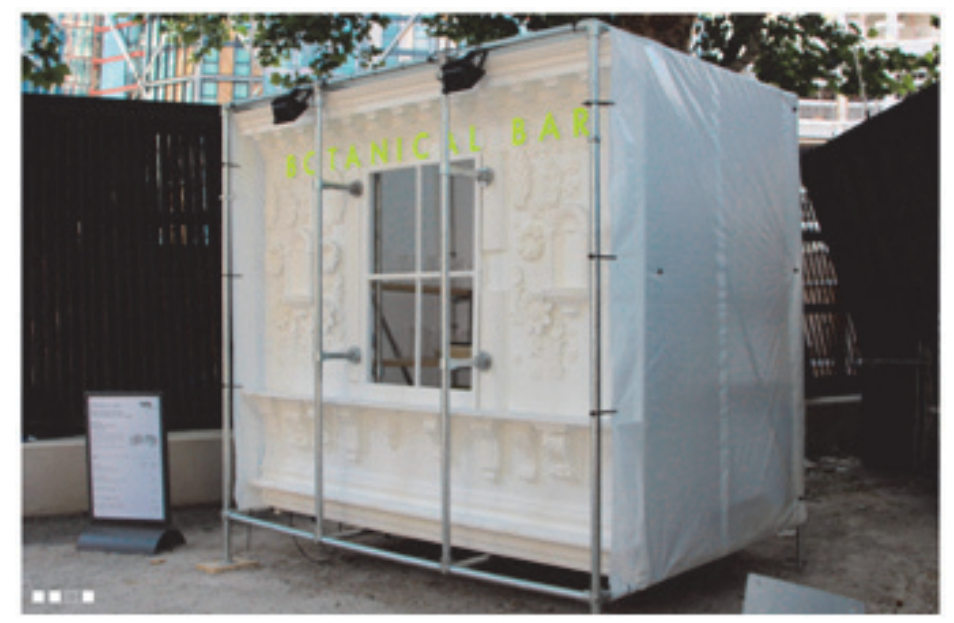

Fig. 3 Future Baroque (Fonte: Something \& Son) 
A eficiência da materialidade. O recurso a ferramentas digitais de simulação e fabricação aditiva na procura de uma maior eficácia dos dispositivos.

A dimensão de prolongamento da vida útil das coisas é também explorada na instalação "Future Baroque" (Fig. 3) que o grupo "Something \& Son" realizou para o museu Tate Modern, em Londres.

O projeto future baroque insere-se numa investigação que procura práticas que permitam incrementar a sustentabilidade ambiental das cidades, respeitando e enriquecendo em simultâneo edifícios antigos que pela desadequação à legislação atual teriam de ser profundamente alterados ou demolidos.

A instalação começa por ser uma fachada ornamentada, cujas formas são obtidas por técnicas tradicionais de moldagem, que são gradualmente substituídas por réplicas impressas. Esta técnica permite que no processo de substituição se coloquem elementos semelhantes, mas muito mais ornamentados do que os anteriormente existentes.

\subsection{Produtos mais leves e eficientes}

Nas técnicas convencionais de produção é frequente configurar os produtos de modo a que, além de suportarem as condições de uso, a sua forma seja adequada a determinada tecnologia de fabricação. Podem ser indicados exemplos como no caso da injeção de plásticos em que a forma do produto deverá permitir a fluência da matéria durante o preenchimento do molde ou situações em que a peça deverá obedecer a determinada geometria para evitar maquinações complexas e ser simples de montar. Os processos de produção aditiva associados à flexibilidade formal permitida pelas ferramentas de modelação e simulação possibilitam a eliminação de moldes e outros acessórios da produção convencional originando peças com as mesmas especificações funcionais e utilizando menos material.

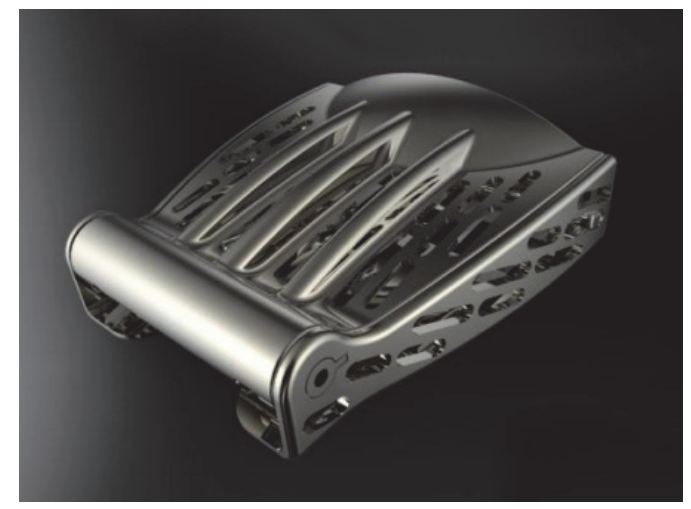

Fig. 4 Otimização da fivela do cinto de segurança (Fonte: Crucible Industrial Design)

A figura 4 apresenta uma fivela do cinto de segurança de um avião otimizada e produzida em titânio com a tecnologia DMLS (Direct Metal Laser Sintering). Este produto fez parte de um projeto ${ }^{70}$ de pesquisa sobre o uso de processos aditivos de produção para reduzir as emissões de carbono. O projeto, inicialmente, segundo os promotores, parecia impossível dado que o DMLS é muito intensivo em energia e, o processo em si não podia ser "verde".

Este é um importante exemplo no sentido de entender que os benefícios ambientais da tecnologia devem ser medidos em relação a todo o ciclo de vida dos produtos assim obtidos.

A fivela de aço produzida por técnicas convencionais pesaria $155 \mathrm{~g}$ enquanto a versão otimizada apenas $70 \mathrm{~g}$ o que equivale a uma redução de $55 \%$ no peso. Para um avião Airbus 380, com 853lugares, a substituição das fivelas corresponderia a uma redução no peso de mais de $70 \mathrm{Kg}$. Durante a vida útil do

\footnotetext{
${ }^{70}$ Os parceiros do projeto foram: Plunkett Associates, Crucible Industrial Design, EOS, 3T PRD, Simpleware, Delcam e University of Exeter
} 
avião a economia em combustível seria mais de 3 milhões de litros. A análise do ciclo de vida, para este caso, resultaria numa redução significativa das emissões de $\mathrm{CO}_{2}$.

É na indústria aeronáutica que se está a investir mais nos processos de produção aditivos complementados pela otimização topológica de projeto. $\mathrm{O}$ método permite uma redução substancial do peso dos componentes garantindo a funcionalidade e resistência estrutural. Nas figuras 5 e 6 (Radny, 2010) apresentam-se as diversas fases do processo de otimização de uma asa de avião e o resultado final.

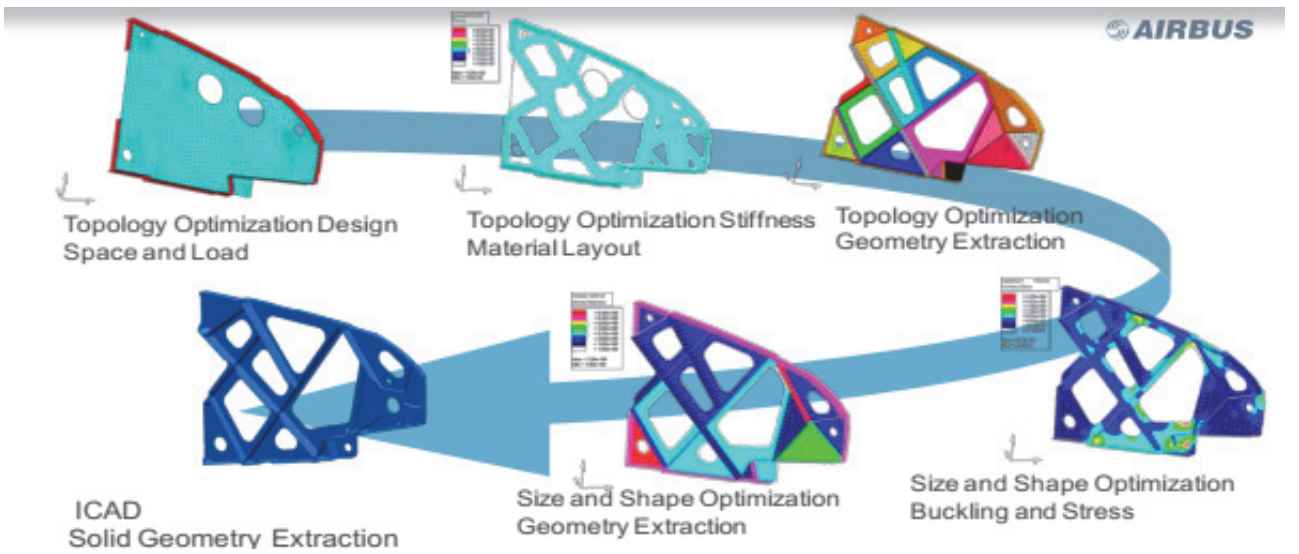

Fig. 5 Otimização topológica de asa de avião (Fonte: AIRBUS)

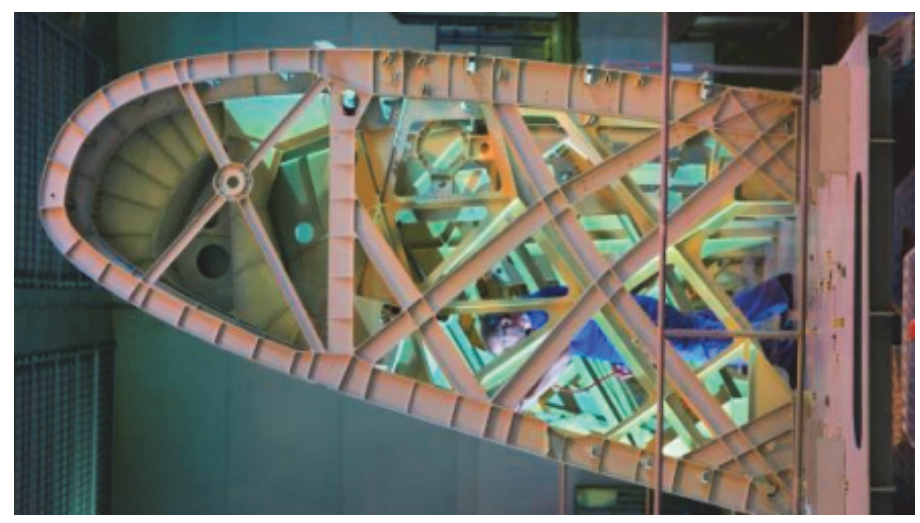

Fig. 6 Componente otimizado (Fonte: AIRBUS)

Outro método de tornar os produtos mais leves é a utilização de estruturas celulares ou em grade (lattice) e malhas. A forma exterior mantém as suas caraterísticas funcionais, ergonómicas ou estéticas, enquanto o interior do objeto é preenchido com uma estrutura mais leve. Na figura 7, apresenta-se um exemplo da indústria automóvel com o veículo conceptual EDAG Light Cocoon com uma estrutura biónica otimizada impressa em 3D. 


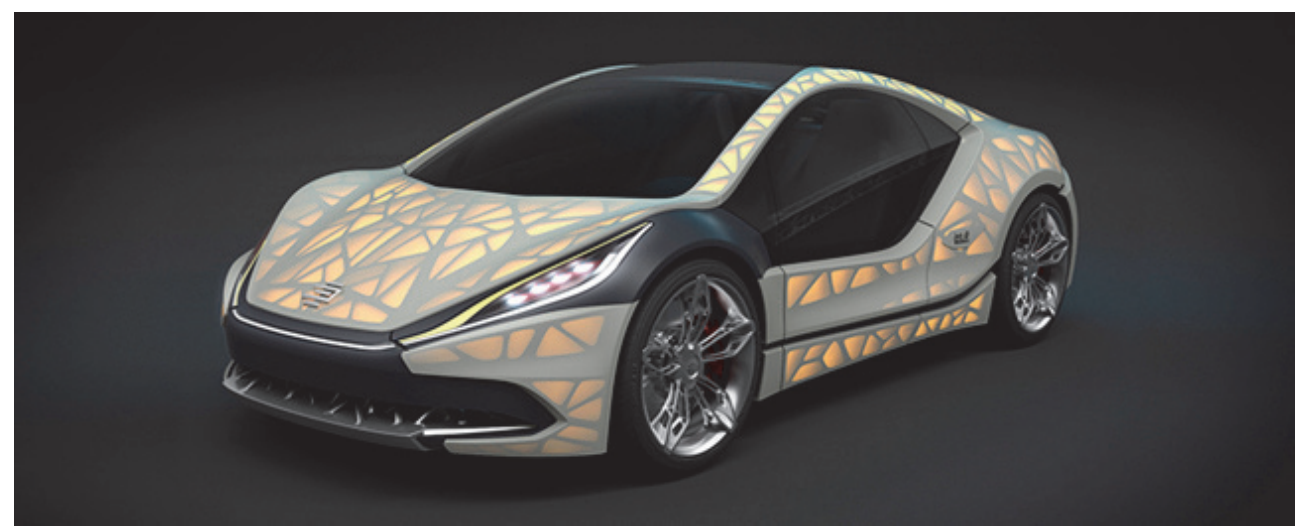

Fig. 7 "EDAG Light Cocoon": the ultimate in lightweight construction (Fonte: (EDAG. 2014)

\subsection{Produção aditiva e sustentabilidade global}

Os processos de produção aditiva representam tecnologia de fabrico relativamente nova, que se carateriza por potenciar fortes estímulos para o desenvolvimento sustentável. Há, no entanto, pouca bibliografia que quantifique objetivamente os benefícios da sua utilização.

Gebler (Gebler, 2014) apresenta um estudo que, segundo o autor, representa a primeira avaliação abrangente destas tecnologias a partir de uma perspetiva global da sustentabilidade desenvolvendo uma avaliação qualitativa das implicações de sustentabilidade induzida e quantifica as variações no ciclo de vida, custos, quantidade de energia e as emissões de $\mathrm{CO} 2$ a nível mundial até 2025. Apesar de Gebler entender que estas tecnologias funcionam ainda como um nicho de aplicação limitado e que, considerando a incerteza da sua utilização noutra escala, concluiu que em 2015, os processos de produção aditiva garantiriam uma redução de $5 \%$ nas emissões de $\mathrm{CO}_{2}$ e no consumo de energia. $\mathrm{O}$ autor refere ainda que se outras indústrias como a automóvel optarem por substituir, mesmo parcialmente, as técnicas convencionais por processos aditivos de produção o potencial (teórico) da redução de emissões e de consumo de energia seria considerável.

\section{Conclusões}

Este trabalho permitiu, através da bibliografia e exemplos referidos, apontar um conjunto de possibilidades das tecnologias de produção aditiva que, em conjunto com o design, permitem encarar de forma positiva a evolução das tecnologias de produção no sentido de uma maior sustentabilidade dos produtos.

Extrapolando a realidade atual para aquilo que pode ser um futuro próximo, as tecnologias aditivas contribuirão fortemente para uma nova forma de pensar o projeto e o desenvolvimento de produtos, muito mais centrada no utilizador e nas suas necessidades e desejos. A capacidade de personalização precisa (já existente na área médica, por exemplo) será possibilitada por uma relação mais próxima entre o designer e o utilizador, potenciada pelas plataformas digitais colaborativas que permitirão a aquisição de dados mais precisos relativos à definição do produto na fase de desenvolvimento, e ao seu desempenho na fase de utilização.

Em termos de sustentabilidade ambiental, a tecnologia de produção aditiva possibilita uma utilização mais precisa e eficiente do material, na medida em que se ultrapassam a generalidade dos 
constrangimentos de forma característicos das tecnologias de produção tradicional, permitindo componentes tecnicamente mais eficientes, e simultaneamente mais leves e com incorporação de menos material. A dispensa de moldes e ferramentas complexas terá também um importante impacto, na medida em que o material necessário para obter um produto é apenas o que o constitui.

O impacto ambiental positivo das tecnologias aditivas residirá também no facto destas permitirem reproduzir eficazmente componentes técnicos de dispositivos existentes, permitindo prolongar a sua vida útil e atrasar por esta via a sua substituição. Poderemos voltar assim a obter, com grande facilidade, peças que se danificaram e que já não se fabricam.

É razoável considerar que existirão, num futuro próximo, locais onde a digitalização e impressão passarão das duas para as três dimensões. Poderemos aí imprimir uma peça da bicicleta dos anos 50 que já não tinha reparação, mas também digitalizar o nosso pé, para os próximos sapatos se adaptarem perfeitamente. Esta realidade permitirá em muitas circunstâncias aproximar o local de fabricação do local de utilização, com importantes consequências em termos ambientais.

Conclui-se ainda que o novo paradigma da produção, entendido como indústria 4.0, ou $4^{\mathrm{a}}$ revolução industrial, implicará uma nova maneira de pensar os produtos, de forma a aproveitar todas as potencialidades da tecnologia no sentido os tornar mais sustentáveis e adaptados às especificidades de cada utilizador.

\section{Referências}

CARSON, R. (2012) Printemps silencieux. Editions Wildproject.

CHANG, T.-C., Wysk, R. A., \& Wang, H.-P. (1998). Computer-Aided Manufacturing (2 ${ }^{\mathrm{a}}$ ed.). New Jersey: Prentice Hall. pp.596-598

CHUA, C. K., LEONG, K. F., \& LIM, C. S. (2005). Rapid prototyping : principles and applications (2nd ed.). New Jersey: : World Scientific. pp. 33-233

Committee F42 on Additive Manufacturing Technologies. ASTM International. $<$ http://www.astm.org/COMMITTEE/F42.htm>. [Consulta: 10 de março de 2016]

COSKUN, A., ZIMMERMAN, J., \& ERBUG, C. Promoting sustainability through behavior change: A review. Design Studies, 41, Part B, 183-204.

CRUCIBLE INDUSTRIAL DESIGN. <http://www.crucibledesign.co.uk/product-design-portfolio/titanium-aircraftseat-buckle.php $>$.[Consulta: 11 de março de 2016]

CSC. (2012). 3D Printing and the Future of Manufacturing. https://assets1.csc.com/innovation/downloads/LEF_20123DPrinting.pdf [Consulta: 15 de abril de 2016]

DIEGEL, O. (2011). Additive manufacturing: the new industrial revolution. Paper presented at the 12th Rapid Design, Prototyping and Manufacturing Conference, Lancaster University, Lancaster, UK.

DIEGEL, O., SINGAMNENI, S., REAY, S., \& WITHELl, A. (2010). Tools for Sustainable Product Design: Additive Manufacturing. Journal of Sustainable Development, 3(3), 68-75.

EDAG. 2014. <http://www.edag.de/en/edag/news-detail/getarticle/News/detail/edag-light-cocoon-the-ultimate-inlightweight-construction.html $>$. [Consulta: 20 de fevereiro de 2016]

GEBLER, M., SCHOOT UITERKAMP, A. J. M., \& VISSER, C. (2014). A global sustainability perspective on 3D printing technologies. Energy Policy, 74, 158-167.

GIBSON, I., ROSEN, D., \& STUCKER, B. (2010). Additive Manufacturing Technologies - Rapid Prototyping to Direct Digital Manufacturing. New York: Springer.

GIBSON, I., ROSEN, D., \& STUCKER, B. (2010). Additive Manufacturing Technologies - Rapid Prototyping to Direct Digital Manufacturing. New York: Springer.p. 287 
A eficiência da materialidade. O recurso a ferramentas digitais de simulação e fabricação aditiva na procura de uma maior eficácia dos dispositivos.

HAGUE, R. (2006). Unlocking the Design Potential of Rapid Manufacturing. In Rapid manufacturing : an industrial revolution for the digital age. England:Chichester: John Wiley.

HAGUE, R. J. M., CAMPBELL, R. I., \& DICKENS, P. M. (2003). Implications on design of rapid manufacturing. Journal of Mechanical Engineering Science, 217(1), 25-30.

HAGUE, R., MANSOUR, S., \& SALEH, N. (2003). Design opportunities with rapid manufacturing. Assembly Automation, 23(4), 346-356.

HOPKINSON, N., HAGUE, R. J. M., \& DICKENS, P. M. (2006). Rapid manufacturing : an industrial revolution for the digital age. Chichester, England: John Wiley. p. 56

LIPSON, H., \& KURMAN, M. (2013). Fabricated: The new world of 3D printing. Indianapolis: John Wiley \& Sons, Inc. p. 11

MALDONADO, T (1972). Design, nature and revolution. New York : Harper \& Row p.4

MARGOLIN, V.(2014). Design e risco de mudança. Vila do Conde: Verso da história

PAPANEK, V.(1974). Design pour um monde réel. Paris: Mercure de France p.260

PIPES, A. (1990). Drawing for 3-Dimensional Design - Concepts - Ilustration - Presentation. London: Thames and Udson. p. 14

RADNY, M. (2010). Additive Layer Manufacturing: A new Perspective in Structural Design . <http://www.3dprinting-additive-manufacturing.com/media/downloads/145-d2-14-30-a-radny-matthias-airbus.pdf $>$. [Consulta: 10 de maio de 2016]

RAWSTHORN, A. (2013). Hello World - where design meets life. London: Penguin Books. pp. 193, 201

SCHODEK, D., BECHTHOLD, M., GRIGGS, J. K., KAO, K., \& STEINBERG, M. (2005). Digital Design and Manufacturing: CAD/CAM Applications in Architecture and Design. New Jersey: Wiley \& Sons. p. 297

SOMETHING \& SON. FUTURE BAROQUE <. http://somethingandson.com/>. [Consulta: 10 de Abril de 2016]

STEGALL, N. (2006). Designing for Sustainability: A Philosophy for Ecologically Intentional Design. Design Issues, 22(2), 56-63.

The Telegraph. < http://www.telegraph.co.uk/news/worldnews/asia/china/12051354/Chinese-buy-up-bottles-offresh-air-from-Canada.html $>$ [Consulta: 16 de Abril de 2016]

Top Ten Toxic Treats 2013. <http://www.worstpolluted.org/projects_reports/display/107>. [Consulta: 15 de abril de 2016]

VEZZOLI, C., \& MANZINI, E. (2008). Design for environmental sustainability London: Springer Science \& Business Media. p.7

VOlpato, N., Ahrens, C., FerReira, C., Petrush, G., CARVAlHO, J., SANTOS, J., ET AL. (2007). Prototipagem Rápida. Tecnologias e Aplicações. S. Paulo - Brasil: Editora Blücher. pp. 55-99

WOHLERS, T. (2011). Wohlers Talk: Views, perspective, and commentary. $<$ http://wohlersassociates.com/blog/2011/08/additive-manufacturing-education/>. [Consulta: 15 de Abril de 2016]

WOHLERS, T. (2015). Wohlers Report: 3D Printing and Additive Manufacturing State of the Industry: Wohlers Associates Inc.

Worstpolluted.org <www.worstpolluted.org/projects_reports/display/107>[Consulta: 10 de Abril de 2016] [sem autoria reconhecida] 